\title{
Magnetic Neutral Points and Electric Lines of Force in Strong Gravity of a Rotating Black Hole
}

\author{
V. Karas, O. Kopáček, D. Kunneriath \\ Astronomical Institute, Academy of Sciences, Prague, Czech Republic \\ Email: vladimir.karas@cuni.cz
}

Received March 9, 2013; revised April 10, 2013; accepted April 19, 2013

Copyright (C) 2013 V. Karas et al. This is an open access article distributed under the Creative Commons Attribution License, which permits unrestricted use, distribution, and reproduction in any medium, provided the original work is properly cited.

\begin{abstract}
Magnetic field can be amplified and twisted near a supermassive black hole residing in a galactic nucleus. At the same time magnetic null points develop near the horizon. We examine a large-scale oblique magnetic field near a rotating (Kerr) black hole as an origin of magnetic layers, where the field direction changes abruptly in the ergosphere region. In consequence of this, magnetic null points can develop by purely geometrical effects of the strong gravitational field and the frame-dragging mechanism. We identify magnetic nulls as possible sites of magnetic reconnection and suggest that particles may be accelerated efficiently by the electric component. The situation we discuss is relevant for starving nuclei of some galaxies which exhibit episodic accretion events, namely, Sagittarius A* black hole in our Galaxy.
\end{abstract}

Keywords: Galaxies: Nuclei; Galaxy: Center; Black Hole Physics; Magnetic Fields

\section{Introduction}

Most galaxies including the Milky Way are believed to host a supermassive black hole in the centre [1,2]. The black hole is embedded in a surrounding gaseous medium and magnetic fields and it can often rotate with an almost extreme value of angular momentum, thereby interacting with magnetic fields of external origin [3]. Electromagnetic processes have been proposed to explain the origin of high-energy particles and radiation flares from black hole (BH) sources. However, there is a variety of possible mechanisms whose details have not yet been fully understood $[1,2,4,6]$. Here we consider the innermost regions where a cooperation of strong gravitational and electromagnetic fields seems to be required [7, $8]$. We study narrow magnetic layers arising by a purely gravitational effect of a rotating BH. These layers are potential sites of acceleration which can operate as the electric field acts across magnetic null points.

We begin this investigation by assuming an organised (ordered) large-scale magnetic field. This is obviously a crude starting point, but a sensible one, representing the field generated by sources distant from the BH (astrophysical black holes are practically uncharged and possess no intrinsic dipole-like magnetic fields). Such a premise about the field structure appears adequate also in the case of Sagittarius $A^{*}\left(\operatorname{Sgr~A}{ }^{*}\right)$, where the black hole of $4 \times 10^{6}$ solar masses resides [6,9]. Given the compact size of the black hole horizon, the magnetic field generated by external sources appears to be effectively uniform on the length-scale a several gravitational radii. However, the field intensity is uncertain. On large scales (i.e., greatly exceeding the gravitational radius) the field should not go beyond a few milligauss $[10,11]$, while on medium scales $\left(10-20 r_{g}\right)$ it might be amplified to tens of gauss $[5,12,13]$.

Magnetic reconnection occurs when the magnetic field lines change their c]onnectivity $[14,15]$. This happens as topologically distinct regions approach each other. The standard setup involves the violation of the ideal MHD approximation just on the boundary between neighbouring magnetic domains where the field direction changes rapidly.

One can ask if the $\mathrm{BH}$ proximity creates conditions favourable to incite reconnection, leading to plasma heating and particle acceleration. This could generate the flaring activity [16-18]. The typical rise time of Sgr A* flares lasts several minutes, i.e. a fraction of the orbital period near the innermost stable circular orbit (ISCO). The variety of processes has been considered for Sgr A* radiation. For example, $[19,20]$ propose that stochastic acceleration of electrons in the turbulent magnetic field is responsible for the submillimeter emission within $20 r_{g}$. 
Also, [21] elaborate on the idea that $\operatorname{Sgr} \mathrm{A}^{*}$ may be an important site for particle acceleration. We will discuss a complementary scheme of a magnetically dominated system.

We show that antiparallel field lines are brought into mutual contact, within the low-density conditions, by the frame-dragging (gravito-magnetic) action of the rotating $\mathrm{BH}$. One expects that a dissipation region develops where the magnetic field structure changes abruptly across a separatrix curve, so these spots, occurring just above the ISCO, can act as places where particles are energised [12]. Ingredients necessary for this scenario to work-i.e. an ordered magnetic field due to external sources plus the diluted plasma environment of disturbed stars-are naturally present near Sgr $\mathrm{A}^{*}$ black hole. In addition to our previous work [22] we show also the electric field threading the magnetic nulls. Thereby the electric component is capable of accelerating the charged matter once it is injected in the area of the magnetic null.

\section{Forming Magnetic Layers by Gravitational Frame-Dragging}

In underluminous galactic nuclei, it is likely that plasma is only episodically injected into the central region, perhaps by passing stars gradually sinking down to the $\mathrm{BH}$. We model the gravitational field by Kerr metric [42]; we will use geometrical units with $c=G=1$ and scale all quantities by the $\mathrm{BH}$ mass, $M$. The gravitational radius is $r_{g}=c^{-2} G M \approx 4.8 \times 10^{-7} M_{7} \mathrm{pc}$, and the corresponding light-crossing time-scale $t_{g}=c^{-3} G M \approx 49 M_{7} \mathrm{sec}$, where $M_{7} \equiv M /\left(10^{7} M_{\odot}\right)^{g}$.

Hereafter we use spheroidal coordinates with $\mu \equiv \cos \theta, \sigma \equiv \sin \theta$, and the metric line element in the form

$$
\begin{aligned}
\mathrm{d} s^{2}= & -\Delta \sum A^{-1} \mathrm{~d} t^{2}+\sum \Delta^{-1} \mathrm{~d} r^{2} \\
& +\sum \mathrm{d} \theta^{2}+A \Sigma^{-1} \sigma^{2}(\mathrm{~d} \phi-\omega \mathrm{d} t)^{2},
\end{aligned}
$$

where $a$ denotes the specific angular momentum, $|a| \leq 1 \quad(a=0$ is for a non-rotating $\mathrm{BH}$, while $a= \pm 1$ is for the maximally co/counter-rotating one),

$\Delta=r^{2}-2 r+a^{2}, \quad \Sigma=r^{2}+a^{2} \mu^{2}$,

$A=\left(r^{2}+a^{2}\right)^{2}-\Delta a^{2} \sigma^{2}$, and $\omega=2 a r / A$. The outer horizon, $r \equiv r_{+}(a)$, is where $\Delta=0$, whereas the ISCO ranges between $r_{+}(a) \leq r_{\text {isco }}(a) \leq 6$. The presence of terms $\propto \omega$ in Kerr metric indicates that frame dragging operates and affects the motion of particles and the structure of fields $[23,24]$.

The influence of general relativistic frame-dragging on accreted particles resembles the effect of a rotating viscous medium: it forces the particles to share the rotational motion of the central body. Similarly, it also affects the magnetic field lines and generates the electric component. For the magnetic field, we might liken the impact of frame-dragging to the Parker spiral, describing the shape of the (rotating) Sun's magnetic field. In a way, the action of the frame-dragging is reminiscent of neutral points arising due to the interaction of the interplanetary magnetic field with the Earth dipole [25], but here the strong gravity plays a major role. Besides other contrasts, the influence of the rotating $\mathrm{BH}$ grows overwhelmingly as one enters the ergospheric region below $r=2 r_{g}$.

The models of electromagnetic acceleration and collimation of jets have been greatly advanced during the last decade [26-29]. These works demonstrate the astrophysical importance of $\mathrm{BH}$ rotation, but their setup is somewhat different from this paper. In particular, the field is typically assumed to be frozen into an equatorial accretion disc; the magnetic field lines are twisted by differential rotation of the disc plasma. On the other hand, the case of $\mathrm{Sgr} \mathrm{A}^{*}$ is distinct, with only a tiny accretion rate, $\dot{M} \leq 10^{-7} M_{\odot} \mathrm{yr}^{-1}[30]$, although any firm estimates are currently not possible because of uncertainties in the accretion efficiency [1]. The standard disc is not present here; the gas chunks arrive episodically [31,32]. In these circumstances one cannot expect the $\mathrm{BH}$ and the ordered magnetic field to have a common symmetry axis (the Bardeen-Petterson effect does not operate due to the lack of a steady accretion flow) neither that the black hole is resting in the centre.

We assume that the electromagnetic field does not contribute to the system gravity, which is correct for every astrophysically realistic situation. Within a limited volume around the $\mathrm{BH}$, typically of size $\simeq\left(10 r_{g}\right)^{3}$, the magnetic field lines have a structure resembling the asymptotically uniform field. The electromagnetic field is a potential one and can be written as a superposition of two parts: the aligned component [33,34], plus the asymptotically perpendicular field $[35,36]$. The fourpotential has been given explicitly in terms of functions $\psi \equiv \phi+a \delta^{-1} \ln \left[\left(r-r_{+}\right) /\left(r-r_{-}\right)\right]$is the Kerr ingoing angular coordinate, $\Psi=r \cos \psi-a \sin \psi, \delta=r_{+}-r_{-}$,

and $r_{ \pm}=1 \pm \sqrt{1-a^{2}} \quad[22,36]$. It is exactly the variable $\psi$ that determines the growing twist of magnetic lines, which eventually leads to the formation of magnetic null points near a rotating black hole. We can thus employ these expressions to draw lines of force.

A particle can be accelerated by the equipartition field, acting along the distance $\ell$, to energy

$\gamma_{\max } \simeq 10^{18} q_{e}(B / 10 G)\left(\ell / r_{g}\right) \mathrm{eV}$, where $q_{e}$ is in units of the elementary charge. Naturally, this rough estimate can be exceeded if a non-stationary field governs the acceleration process.

The aligned vacuum field is gradually expelled out of the $\mathrm{BH}$ as its rotation increases [33]. Non-vacuum fields are more complicated and have been studied in detail; e.g. [37] notice that high conductivity of the medium changes 
the field expulsion properties of the horizon. On the other hand, only few aspects of the misaligned $\mathrm{BH}$ magnetic fields have been explored so far $[38,39]$. This is because of an extra complexity caused by the frame-dragging $[40,41]$. What we put forward here is that the framedragging can actually contribute to the acceleration mechanisms.

To obtain the physical components of the electromagnetic tensor, $F=2 \mathrm{~d} A$, we project it onto the local observer tetrad, $\mathrm{e}_{(a)}$ [42]. The appropriate choice of the projection tetrad is the one attached to a frame in Keplerian orbital motion, which exists for $r \geq r_{\text {isco }}$. Free circular motion can be stable at $r \geq r_{\text {isco }}$, otherwise the star has to spiral downwards while maintaining constant angular momentum of $l=l\left(r_{\text {isco }}\right)$. The corresponding frame and the field lines can be derived in a straightforward manner [22]. We interpret the frame, quite naturally, as the one connected with an orbiting star. In the extremely rotating case, the circular motion of a star is possible all the way down to $r_{\text {isco }}=r_{g}$. The dependence of the electromagnetic components on the $\psi$ angle then indicates the ever increasing effect of the frame dragging near the horizon.

The electric and magnetic intensities, measured by the physical observer, are:

$$
E_{(a)}=e_{(a)}^{\mu} F_{\mu \nu} u^{v}, B_{(a)}=\mathrm{e}_{(a)}^{\mu} F_{\mu \nu}^{*} u^{v},
$$

where $u^{v} \equiv \mathrm{e}_{(t)}^{v}$ is the observer's four-velocity (the remaining three basis vectors can be conveniently chosen as space-like, mutually perpendicular vectors).

Figure 1 shows the typical structure of the field lines representing an asymptotically transverse magnetic field $(B=0)$. Formation of the layers in the ergospheric region just above ISCO is an interesting and generic feature of the rotating spacetime. Two critical points can be seen occurring at radii up to $\simeq 1.7 r_{g}$ for $a=1$. Naturally, the co-rotating case $(a>0)$ is not equivalent with the counter-rotating $(a<0)$ one, but in both, a site of oppositely directed field lines arises.

An example of electric lines of force is plotted in the right panel of Figure 1. We notice that the electric field along the magnetic lines does not vanish and is capable of accelerating charged particles. Figure 2 then reveals the shape of the near-horizon field lines in terms of the new radial variable, $r^{*} \equiv 1-r_{g} / r$, which we introduce to better resolve the complicated structure (Cartesian-type coordinates are used, $x^{* 2}+y^{* 2}=r^{* 2}$ ).

So far we have assumed zero translational motion of the $\mathrm{BH}$ with respect to the magnetic field. However, our approach can be generalised and the uniform motion can be taken into account by Lorentz boost of the field intensities $E_{(a)}, B_{(a)}$. To this end we notice that the Lorentz transform, when applied in the asymptotically distant region (i) changes the direction of the uniform
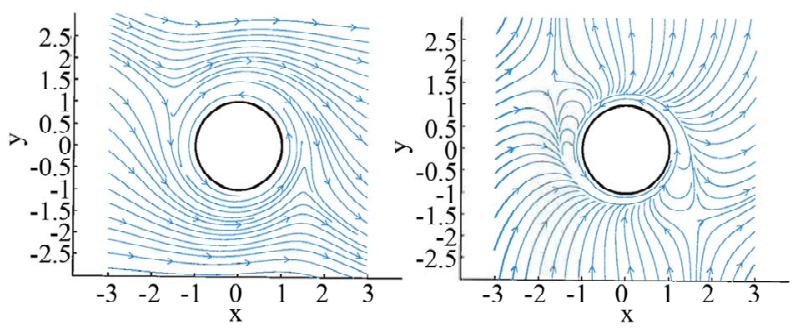

Figure 1. Magnetic field lines lying in the equatorial plane $x y$, perpendicular to the rotation axis of an extremely rotating BH (units are scaled with of gravitational radius $r_{g}$ ). Left panel: an asymptotically uniform magnetic field is directed along the $x$-axis at large radii and plotted with respect to the physical frame of an orbiting star. The case of a co-rotating frame is shown $(a=1)$. Right panel: example of the projection of the rotation induced electric field, corresponding to the magnetic field in the left panel.
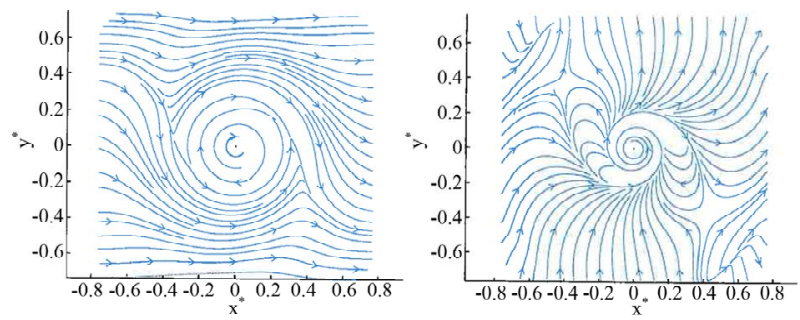

Figure 2. The field structure as in the left panel of Fig. 2, now using the new radial coordinate $r^{*}$. The magnetic field (left panel) and the corresponding electric field (right panel) are shown.

magnetic field by an angle $\vartheta$, and (ii) generates a new (uniform) electric component. The desired form of the electromagnetic test field near a moving black hole is thus written in a symbolic way, $F_{r \rightarrow \infty}^{\prime}=\Lambda(\beta)^{\mathrm{T}} F \Lambda(\beta)$, and obtained as superposition of the two parts combined together in the right ratio, i.e. the asymptotically uniform magnetic field, rotated into the desired direction, and the solution for the asymptotically uniform electric field. The latter one can be found by applying the dual transform to Wald's field.

This way we can explore also the magnetic fields near a drifting $\mathrm{BH}$, in which case the additional electric component arises. As an example, in the right panel of Figure 2 we assumed constant velocity $\beta_{y}=-0.99$ directed along the $y$-axis. A null point (again shown in the orbiting frame) arises just above ISCO, located at $x^{2}+y^{2}=1$ for $a=1$. Finally, Figure 3 shows an enlarged detail of the magnetic structure around the region where the magnetic null point develops. The two examples differ by the magnitude of the translatory boost of the black hole which affects the exact location of the null point, although it always appears very close to the horizon and requires rapid rotation of the black hole.

Finally, we remind the reader that the shape of lines of force obviously depends on the observer's frame with 

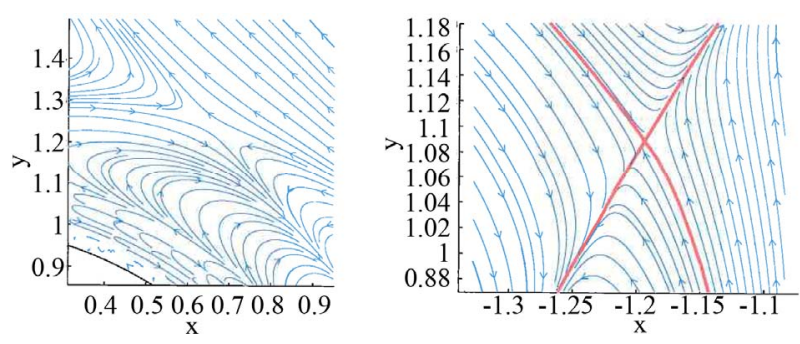

Figure 3. Two examples of detailed magnetic structure surrounding a null point, where the magnetic field vanishes in the black hole equatorial plane. The separatrix line is shown (solid line by red colour) in the right panel.

respect to which the lines are plotted. Figure 4 compares three distinct examples. The there panels of the top row present the lines of magnetic force with respect to the free-falling tetrad (observer's angular momentum vanishes). Further down, the second row corresponds to the case of a free-falling observer (below the innermost stable circular orbit) in combination with a freely orbiting observer (Keplerian prograde rotation) above it; likewise in the third row (for retrograde rotation). The Schwarzschild limit $a=0$ is shown in the first column, middle column corresponds to spin $a=0.9$, and the last one represents the extreme case, $a=1$. In the bottom row the rescaled dimensionless radial coordinate

$R \equiv r-r_{+} / r$ is used in order to stretch the region close to the horizon (case of extreme rotation).

Complementary to magnetic field lines, Figure 5 shows the projection of electric lines of force induced by the presence of the magnetic component by rotation and the linear motion of the black hole. The first (left) column presents the non-drifting case (zero boost velocity $v_{i}$ ); in the second we set $v_{x}=0.5, v_{y}=0$; and in the third $v_{x}=0.5, v_{y}=0.5$. Four distinct cases are compared in the rows (top to bottom): zero-angular momentum observers, free-falling observers, co-rotating and free falling, and counter-rotating and free-falling observers, respectively. We observe that in all these cases the neutral electric points develop as the drift is introduced. We stress that as the original field is aligned $\left(B_{x}=0\right) \quad$ we measure the latitudinal component $E^{(\theta)}=0$ in the equatorial plane provided that $v_{z}=0$, so actually these plots present true shape of field lines.

We notice that non-vanishing electric component passes through the magnetic null, thereby accelerating electrically charged particles in this region. One expects reconnection to occur intermittently, as the plasma is injected into the dissipation region where the differently directed field lines approach each other due to their interaction with a highly curved spacetime. Will the gravito-magnetic effect produce the same layered structure of the magnetic field also in the presence of non-negligible amount of plasma, or will the field structure change entirely? Numerical simulations will be necessary to see whether this mechanism can be part of a broader picture in astrophysically realistic situations, which vary wildly under different circumstances, and to determine the actual speed at which the process operates. We remark that, on the other end of analytical approximations, the solution for non-axisymmetric accretion of stiff adiabatic gas onto a rotating black hole also exhibits critical points near the horizon [43], so the conditions for magnetic reconnection of the frozen-in magnetic field will be again fulfilled.

\section{Conclusions}

We considered the influence of the black hole rotation acting onto the ordered magnetic field in the physical frame of a star orbiting a black hole, or plunging down to it. If rotation is fast enough, the magnetic layers and the corresponding null points exist just above the innermost stable circular orbit. Although we prescribed a special configuration of the electro-vacuum magnetic field and we considered only the test-field approximation, the process of warping the field lines is a general feature that should operate also in more complicated settings: the frame-dragging is expected to take over and determine the field structure near the horizon. The layered structure of the magnetic field in lines with neutral points suggests that should become a site of particle acceleration.

The essential ingredients of the scheme described here are the rotating black hole and the oblique magnetic field into which the black hole is embedded. The interaction region is very near the horizon, representing, to our knowledge, the acceleration site nearest to the black hole horizon among the variety of mechanisms proposed so far. Even if we treated the problem in a very simplified scheme, the idea of geometrical effects of frame dragging causing the acceleration of matter is very promising in the context of rapidly rotating black hole inside nuclei of galaxis.

We concentrated on the equatorial plane in which the transverse magnetic field lines reside, but this constrain was imposed only to keep graphs as clean as possible. Otherwise, the lack of symmetry complicates the situation. Plasma motions in the close vicinity of the Sgr A* black hole are currently inaccessible to direct observation. However, there are chances that the region will be resolved with future interferometers, such as GRAVITY in the near-infrared spectral band and the Event Horizon Telescope VLBI project in submillimeter wavelengths.

\section{Acknowledgements}

We acknowledge the Czech Science Foundation (GAČr 13-00070J) and German Forschungsgemeinschaft (DFG) 

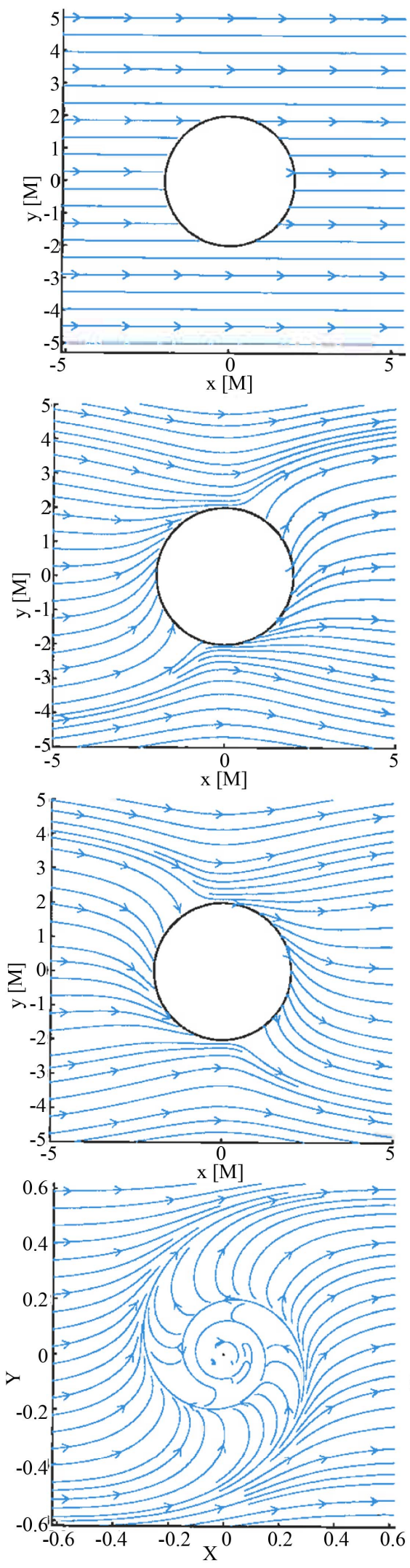
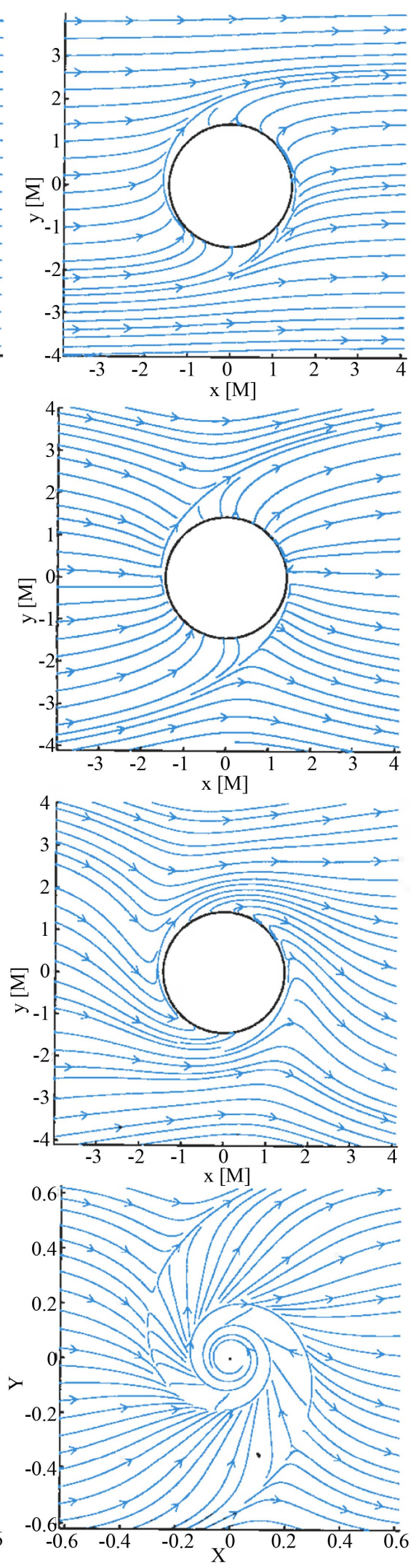
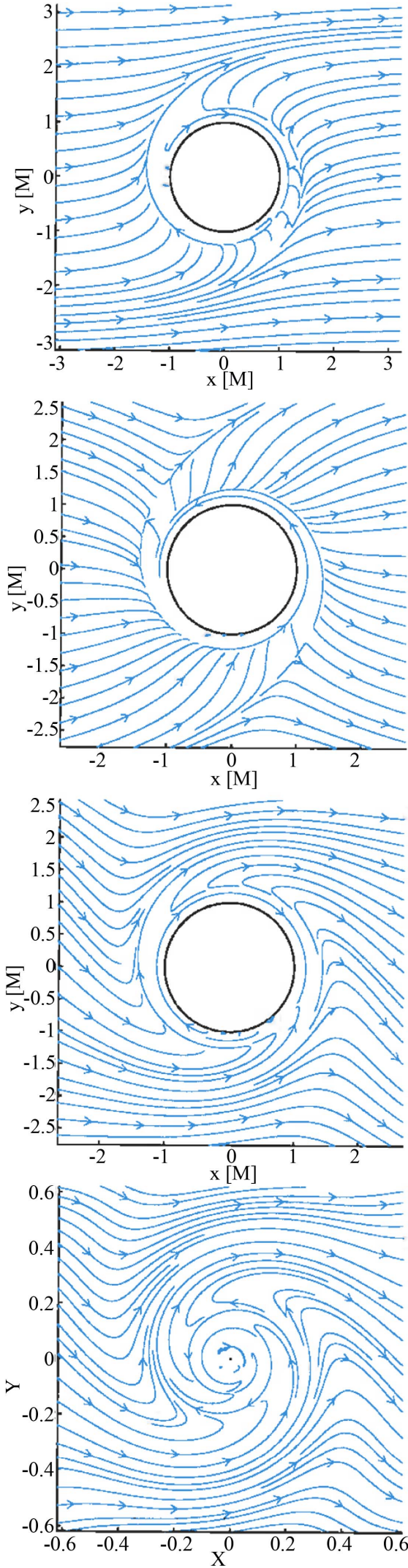

Figure 4. Equatorial behaviour of the magnetic field with the purely perpendicular magnetic asymptotes $\left(B_{x} \neq 0, B_{y}=B_{z}=0\right.$, for three distinct tetrads (see the main text for details). 

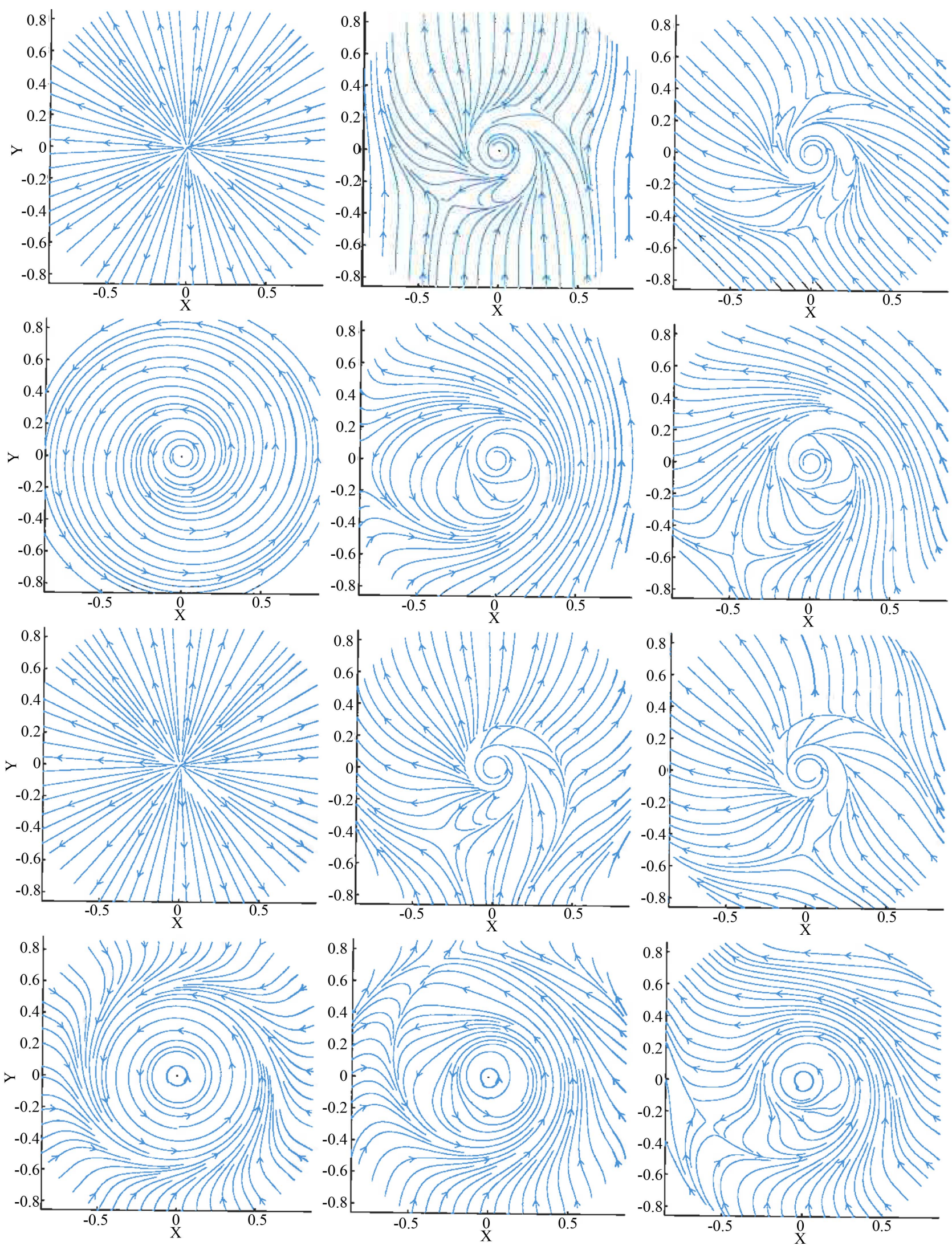

Figure 5. Equatorial behaviour of the electric field projection. Case of the extreme Kerr black hole drifting with respect to the aligned magnetic field. 
collaboration project for support.

\section{REFERENCES}

[1] A. Eckart, R. Schödel and C. Straubmeier, "The Black Hole at the Center of the Milky Way," Imperial College Press, London, 2005.

[2] F. Melia, "The Galactic Supermassive Black Hole," Princeton University Press, Princeton, 2007.

[3] R. Beck and R. Wielebinski, "Magnetic Fields in Galaxies, in Planets, Stars and Stellar Systems," In: T. D. Oswalt and G. Gilmore, Eds., Springer, Vol. 5, 2013. arXiv:1302.5663

[4] M. C. Begelman, R. D. Blandford and M. Rees, Reviews of Modern Physics, Vol. 56, 1984, p. 255. doi:10.1103/RevModPhys.56.255

[5] A. Eckart, F. K. Baganoff, M. Zamaninasab, et al., Astronomy \& Astrophysics, Vol. 479, 2008, p. 625.

[6] R. Genzel, F. Eisenhauer and S. Gillessen, Reviews of Modern Physics, Vol. 82, 2010, p. 3121. doi:10.1103/RevModPhys.82.3121

[7] E. Boldt and P. Ghosh, Monthly Notices of the Royal Astronomical Society, Vol. 307, 1999, p. 491. doi:10.1046/j.1365-8711.1999.02600.x

[8] A. Levinson, Physical Review Letters, Vol. 85, 2000, p. 912. doi:10.1103/PhysRevLett.85.912

[9] M. Morris, Journal of Physics: Conference Series, Vol. 54, 2006, p. 1. doi:10.1088/1742-6596/54/1/001

[10] M. Morris, K. Uchida and T. Do, Nature, Vol. 440, 2006, p. 308. doi: $10.1038 /$ nature 04554

[11] M. P. Muno, F. K. Baganoff, W. N. Brandt, M. R. Morris and J.-L. Starck, Astrophysical Journal, Vol. 673, 2008, p. 251. doi: $10.1086 / 521641$

[12] W. J. Duschl and H. Lesch, Astronomy \& Astrophysics, Vol. 286, 1994, p. 431.

[13] S. Markoff, H. Falcke, F. Yuan and P. L. Biermann, Astronomy \& Astrophysics, Vol. 379, 2001, p. L13.

[14] E. Priest and T. Forbes, "Magnetic Reconnection," Cambridge University Press, Cambridge, 2000,

[15] B. V. Somov, "Plasma Astrophysics," Astr. Sp. Sci. Lib., Vol. 341, Springer, New York, 2006.

[16] F. K. Baganoff, M. W. Bautz, W. N. Brandt, et al., Nature, Vol. 413, 2001, p. 45. doi:10.1038/35092510

[17] R. Genzel, R. Schödel, T. Ott, et al., Nature, Vol. 425, 2003, p. 934. doi: $10.1038 /$ nature02065

[18] Porquet, D., Predehl, P., Aschenbach, B., et al., Astronomy \& Astrophysics, Vol. 407, 2003, p. L17. doi:10.1051/0004-6361:20030983

[19] S. Liu, F. Melia and V. Petrosian, Astrophysical Journal, Vol. 636, 2006, p. 798. doi:10.1086/498128

[20] S. Liu, F. Melia, V. Petrosian and M. Fatuzzo, Astrophysical Journal, Vol. 647, 2006, p. 1099. doi:10.1086/505171

[21] D. R. Ballantyne, F. Melia, S. Liu and R. M. Crocker,
Astrophysical Journal Letters, Vol. 657, 2007, p. L13. doi:10.1086/513103

[22] V. Karas, O. Kopáček and D. Kunneriath, Classical and Quantum Gravity, Vol. 29, 2012, Article ID: 035010. doi:10.1088/0264-9381/29/3/035010

[23] V. Karas and D. Vokrouhlický, Astrophysical Journal, Vol. 422, 1994, p. 208. doi:10.1086/173719

[24] A. Merloni, M. Vietri, L. Stella and D. Bini, Monthly Notices of the Royal Astronomical Society, Vol. 304, 1999, p. 155.

[25] J. W. Dungey, Physical Review Letters, Vol. 6, 1961, p. 47. doi:10.1103/PhysRevLett.6.47

[26] C. Fendt and J. Greiner, Astronomy \& Astrophysics, Vol. 369, 2001, p. 308. doi:10.1051/0004-6361:20010108

[27] S. Koide, Astrophysical Journal Letters, Vol. 606, 2004, p. L45. doi:10.1086/420976

[28] S. S. Komissarov, M. V. Barkov, N. Vlahakis and A. Königl, Monthly Notices of the Royal Astronomical Society, Vol. 380, 2007, p. 51. doi:10.1111/j.1365-2966.2007.12050.x

[29] J. H. Krolik, J. F. Hawley and S. Hirose, Astrophysical Journal, Vol. 622, 2005, p. 1008. doi:10.1086/427932

[30] S. Markoff and H. Falcke, AN, Vol. 324, 2003, p. 445.

[31] R. F. Coker and F. Melia, Astrophysical Journal Letters, Vol. 488, 1997, p. L149. doi:10.1086/310925

[32] J. Cuadra, S. Nayakshin and F. Martins, Monthly Notices of the Royal Astronomical Society, Vol. 383, 2008, p. 458.

[33] A. R. King, W. Kundt and J. P. Lasota, Physical Review D, Vol. 12, 1975, p. 3037. doi:10.1103/PhysRevD.12.3037

[34] R. M. Wald, Physical Review D, Vol. 10, 1974, p. 1680. doi:10.1103/PhysRevD.10.1680

[35] J. Bičák, and L. Dvořák, General Relativity and Gravitation, Vol. 7, 1976, p. 959. doi:10.1007/BF00766421

[36] J. Bičák and V. Janiš, Monthly Notices of the Royal Astronomical Society, Vol. 212, 1980, p. 899.

[37] S. S. Komissarov and J. C. McKinney, Monthly Notices of the Royal Astronomical Society, Vol. 377, 2007, p. L49.

[38] A. Neronov and F. A. Aharonian, Astrophysical Journal, Vol. 671, 2007, p. 85. doi:10.1086/522199

[39] A. Tomimatsu, Astrophysical Journal, Vol. 528, 2000, p. 972. doi: $10.1086 / 308190$

[40] A. N. Aliev and D. V. Galtsov, Soviet Physics, Vol. 32, 1989, p. 75. doi:10.1070/PU1989v032n01ABEH002677

[41] V. Karas, Physical Review D, Vol. 40, 1989, p. 2121. doi:10.1103/PhysRevD.40.2121

[42] C. W. Misner, K. S. Thorne and J. A. Wheeler, "Gravitation,” W. H. Freeman \& Co, New York, 1973.

[43] L. I. Petrich, S. L. Shapiro and S. A. Teukolsky, Physical Review Letters, Vol. 60, 1988, p. 1781. doi:10.1103/PhysRevLett.60.1781 